\title{
MAS in Psychotraumatology: ein innovativer Studiengang an der Universität Zürich
}

Ulrich Schnyder, Robert M. McShine

Korrespondenz:

Prof. Dr. med. Ulrich Schnyder

Facharzt für Psychiatrie

und Psychotherapie FMH

Psychiatrische Poliklinik

Universitätsspital

Culmannstrasse 8

CH-8091 Zürich

Tel. 0442555251

Fax 0442554408

ulrich.schnyder@psyp.uzh.ch

www.psychiatrie.usz.ch

www.mas-psychotraumatology. uzh.ch

\section{Einleitung}

Über die Hälfte aller Menschen macht im Verlauf ihres Lebens mindestens einmal eine traumatische Erfahrung [1, 2]. Dazu gehören schwere Unfälle, technische und Naturkatastrophen, Krieg, Folter, terroristische Gewalt, Überfälle, Kriegsgefangenschaft und Gefangenschaft in einem Konzentrationslager, physische und sexuelle Gewalt, der plötzliche oder gewaltsame Tod einer nahestehenden Person oder die Diagnose einer lebensbedrohlichen Krankheit [3].

Angesichts der Häufigkeit traumatischer Ereignisse erstaunt es nicht, dass die posttraumatischen psychischen Störungen zu den häufigen psychischen Erkrankungen gehören. Die Lebenszeitprävalenz der posttraumatischen Belastungsstörung (PTBS) liegt in der amerikanischen Bevölkerung bei etwa $8 \%[1,2]$, in Australien und den Niederlanden wird über vergleichbare Zahlen berichtet. In der Schweiz scheint die PTBS seltener vorzukommen [4]. Nach interpersonellen Traumatisierungen wie Folter [5, 6] oder Vergewaltigung $[7,8]$ werden allerdings in bis zu $50 \%$ posttraumatische Belastungsstörungen diagnostiziert.

Die PTBS ist nicht nur eine psychologische, sondern auch eine neurobiologische Erkrankung. Das zeigt sich zum Beispiel in einer Beeinträchtigung der Hypothalamus-Hypophysen-Nebennierenrinden-Achse (hypothalamic-pituitaryadrenal axis, HPA-Achse). Personen, die später eine PTBS entwickeln, weisen bereits während bzw. kurz nach dem Trauma tiefe Cortisolwerte auf. Dazu passend weisen erste Forschungsergebnisse darauf hin, dass die Gabe von niedrigen Cortisoldosen zu einer Reduktion der PTBS-Symptomatik führen kann [9].

Der Zusammenhang zwischen Trauma und körperlichen Gesundheitsstörungen wurde erst in den letzten Jahren in seinem vollen Ausmass bekannt: Je mehr traumatischen Erfahrungen eine Person in der Kindheit ausgesetzt war, desto grösser ist ihr Risiko für körperliche Erkrankungen (z.B. Lungen-, Herz-Kreislauf- oder Tumorerkrankungen) im Erwachsenenalter. Patienten mit PTBS haben generell ein erhöhtes Risiko für somatische Morbidität und Mortalität. Man geht

\section{Master of Advanced Studies}

(MAS) en psychotraumatologie: cursus innovateur à l'Université

\section{de Zurich}

L'Université de Zurich est la première en Europe à offrir dès l'automne 2007 un programme d'études accompagnateur pour les psychiatres expérimentés dans le domaine clinique et les psychothérapeutes qui souhaitent parfaire leurs connaissances et aptitudes dans le domaine de la psychotraumatologie. L'accent sera mis sur la procédure thérapeutique basée sur l'évidence scientifique. L'étude dure quatre semestres et est couronnée par un «Master of Advanced Studies in Psychotraumatology». L'offre s'adresse à des professionnels ayant terminé leurs études en médecine humaine ou psychologie clinique. Deux ans au moins d'expérience professionnelle auprès de patients malades psychiques et des études poussées en psychothérapie sont exigées. La combinaison de sessions où la présence est bienvenue avec l'apprentissage par ordinateur (blended learning) permettra d'apprendre de manière asynchrone et donc d'accepter les étudiants de I'ensemble de l'espace germanophone. Vous trouvez d'autres informations sur la page internet de ce cursus: www.mas-psycho traumatology.uzh.ch.

davon aus, dass hier ein komplexes Wechselspiel zwischen psychologischen Faktoren (Depressivität, Feindseligkeit, Coping), gesundheitsrelevantem Verhalten (z. B. Rauchen, Ernährung, Bewegung) und biologischen Prozessen (u. a. kardiovaskuläre Reaktivität, autonomes Hyperarousal, gestörte Schlafphysiologie, adrenerge Dysregulation, erhöhte Schilddrüsenfunktion und veränderte HPA-Aktivität) zum Tragen kommt [10]. 
Seit 1980 wurden verschiedene psychotherapeutische Ansätze zur Behandlung der PTBS entwickelt. In erster Linie für die kognitive Verhaltenstherapie, insbesondere "prolonged exposure» [11] und «eye movement desensitization and reprocessing» [12], konnte eine sehr gute Wirksamkeit gezeigt werden. Das Feld ist jedoch noch sehr in Bewegung, in den nächsten Jahren werden weitere Entwicklungen erwartet [13]. So scheinen beispielsweise die «brief eclectic psychotherapy» [14] und die "narrative exposure therapy» [15] interessante Neuerungen zu bringen. Gute wissenschaftliche Evidenz liegt allerdings bis heute nur für die Behandlung von Menschen mit sogenannten Typ-I-Traumata (einmalig, plötzlich, z. B. Unfall) vor. Hauptsächlich aufgrund der Komplexität der Folgen von sogenannten Typ-II-Traumata (repetitiv, sequentiell, z. B. Folter oder sexueller Missbrauch in der Kindheit) ist die verfügbare wissenschaftliche Evidenz in diesem Bereich noch nicht befriedigend. Aber auch hier wurden therapeutische Ansätze entwickelt, die sich von einer konventionellen Psychotherapie unterscheiden [16, 17]. Bis heute gibt es allerdings weder in der Schweiz noch sonstwo in Europa eine Ausbildung auf universitärem Niveau, in der die Grundlagen der Psychotraumatologie und die wichtigsten traumatherapeutischen Verfahren schulenübergreifend vermittelt werden.

\section{Der Masterstudiengang in Psychotraumatologie}

\section{Inhalt des Studienganges}

Erstmals in Europa wird nun ab Herbst 2007 in Zürich ein berufsbegleitender, schulenübergreifender universitärer Studiengang in Psychotraumatologie angeboten. Der Studiengang vermittelt Kenntnisse und Fertigkeiten im Bereich der Psychotraumatologie, und zwar wo immer möglich auf der Basis der gegenwärtig verfügbaren wissenschaftlichen Evidenz. Dazu gehören die allgemeine und spezielle Psychotraumatologie sowie insbesondere die wichtigsten traumatherapeutischen Ansätze.

Die allgemeine Psychotraumatologie umfasst unter anderem die Geschichte, Epidemiologie und Diagnostik der posttraumatischen Störungen sowie die psychologischen und neurobiologischen Grundlagen der Psychotraumatologie. In der speziellen Psychotraumatologie werden Themen wie Komorbidität, forensische Psychotraumatologie, Salutogenese, spezielle Betroffenengruppen, Täter, «vicarious traumatization», «false memory syndrome», Übertragung und Gegenübertragung behandelt.
Hauptsächlich werden die Studierenden aber die wichtigsten traumatherapeutischen Verfahren in Theorie und Praxis kennenlernen. Dazu gehören Interventionsformen nach akuter Traumatisierung, vor allem aber die wichtigsten Therapieansätze zur Behandlung der posttraumatischen Belastungsstörung, insbesondere «prolonged exposure», «eye movement desensitization and reprocessing», «brief eclectic psychotherapy for PTSD», «narrative exposure therapy» sowie Modelle zur Behandlung komplexer Traumatisierungen. Auch die Prävention und Behandlung bei Helfern und die Psychopharmakotherapie sollen thematisiert werden. Mit Falldarstellungen und Gruppensupervisionen werden die Studierenden ihre therapeutischen Erfahrungen mit diesen Methoden überprüfen können.

\section{Kurskonzept, Lernformen}

Der Masterstudiengang richtet sich an Fachleute mit einem abgeschlossenen Studium in Humanmedizin oder klinischer Psychologie, zudem werden mindestens zwei Jahre Berufserfahrung mit psychisch kranken Menschen und eine fortgeschrittene Psychotherapieausbildung vorausgesetzt. Ziel des Studienganges ist der Erwerb des «Master of Advanced Studies in Psychotraumatology». Der Studiengang dauert 4 Semester und wird berufsbegleitend angeboten. Für den Masterabschluss werden eine Masterarbeit vorgelegt und ein Abschlusskolloquium absolviert. Das Masterdiplom kann nach Erreichen von insgesamt 60 ECTS erworben werden.

Der Studiengang verwendet das Konzept des «blended learning»: E-Learning wird mit Präsenzveranstaltungen kombiniert. Die Vermittlung von Basiswissen wird mit E-Learning unterstützt. So können sich die Teilnehmer im Hinblick auf die Präsenzveranstaltungen auf einen ähnlichen Wissensstand bringen. Die Lernmaterialien werden auf einer Internetplattform zur Verfügung gestellt. Damit besteht die Möglichkeit für ortsunabhängiges und asynchrones Lernen, für virtuelle Gruppenarbeiten und für einen kontinuierlichen Austausch mit dem E-Learning-Moderator.

In den Präsenzveranstaltungen soll das Erarbeitete vertieft werden. Es sind insgesamt etwa 18 zweitägige Präsenzveranstaltungen vorgesehen, die jeweils an einem Freitag und Samstag im Zentrum für Weiterbildung der Universität Zürich stattfinden werden. Die Studierenden werden hier auch prominente Persönlichkeiten der Psychotraumatologie persönlich oder mittels Videokonferenz kennenlernen. 


\section{Programmleitung}

Die Trägerschaft dieses Masterstudiengangs liegt bei der Medizinischen Fakultät der Universität Zürich. Prof. Dr. med. Ulrich Schnyder, Direktor der Psychiatrischen Poliklinik des Universitätsspitals Zürich, hat die akademische Leitung inne. Dr. med. Robert M. McShine MHA, stellvertretender Ärztlicher Direktor des Psychiatriezentrums Hard Embrach, ist für das Projektmanagement zuständig. Frau lic. phil. Regula Flury ist als Programmdirektorin verantwortlich für die Planung, Koordination und Durchführung des Studiengangs.

Der Leitende Ausschuss des Studiengangs setzt sich aus folgenden Fachpersonen aus dem deutschen Sprachraum zusammen: Prof. Dr. med. Ulrich Schnyder (Präsident), Zürich; Prof. Dr. rer. soc. Thomas Elbert, Konstanz; Dr. med. Georges Kopp, Bellikon; PD Dr. phil. Markus Landolt, Zürich; Prof. Dr. phil. Dr. med. Andreas Maercker, Zürich; Dr. med. Robert M. McShine MHA, Embrach; Dr. med. Lina Steinacher, Zürich; Prof. Dr. med. Roland von Känel, Bern; Prof. Dr. phil. Hansjörg Znoj, Bern.

Zudem haben wir eine Zusammenarbeit mit international renommierten Experten aufgebaut: Das International Advisory Board besteht aus folgenden Fachleuten: Prof. Edna B. Foa, PhD, Philadelphia, USA; Prof. Matthew J. Friedman, MD, PhD, White River Junction, VT, USA; Prof. Berthold P. R. Gersons, MD, PhD, Amsterdam, Niederlande; Prof. Alexander McFarlane, MD, Adelaide, Australien; Dr. med. Luise Reddemann, Kall, Deutschland; Prof. Paula P. Schnurr, PhD, White River Junction, VT, USA; Prof. Arieh Y. Shalev, MD, Jerusalem, Israel; Dr. Stuart Turner, London, Grossbritannien.

\section{Anmeldung}

Der Studiengang beginnt am 1. Oktober 2007. Anmeldungen werden bis 30. Juni 2007 entgegengenommen. Weitere Informationen und Kontaktadressen finden sich auf der Website des Studienganges: www.mas-psychotraumatology. uzh.ch.

\section{Literatur}

1 Breslau N, Kessler RC, Chilcoat HD, Schultz LR, Davis GC, Andreski P. Trauma and posttraumatic stress disorder in the community - The 1996 Detroit Area Survey of Trauma. Arch Gen Psychiatry. 1998;55:626-32.

2 Kessler RC, Sonnega A, Bromet E, Hughes M, Nelson CB. Posttraumatic stress disorder in the national comorbidity study. Arch Gen Psychiatry. 1995;52:1048-60.
3 American Psychiatric Association. Diagnostic and Statistical Manual of Mental Disorders. 4th edition. Washington, DC: APA; 1994.

4 Hepp U, Gamma A, Milos G, Eich D, Ajdacic-Gross V, Roessler W, et al. Prevalence of exposure to potentially traumatic events and PTSD: the Zurich Cohort Study. Eur Arch Psychiatry Clin Neurosci. 2006;256:151-8.

5 Basoglu M, Paker M, Paker O, Ozmen E, Marks I, Incesu C, et al. Psychological effects of torture: a comparison of tortured with nontortured political activists in Turkey. Am J Psychiatry. 1994;151:76-81.

6 van Velsen C, Gorst-Unsworth C, Turner S. Survivors of torture and organized violence: demography and diagnosis. J Trauma Stress. 1996;9:181-93.

7 Resnick HS, Kilpatrick DG, Dansky BS, Saunders BE, Best CL. Prevalence of civilian trauma and posttraumatic stress disorder in a representative national sample of women. J Consult Clin Psychol. 1993;61:984-91.

8 Winfield I, George LK, Swartz M, Blazer DG. Sexual assault and psychiatric disorders among a community sample of women. Am J Psychiatry. 1990;147:335-41.

9 Aerni A, Traber R, Hock C, Roozendaal B, Schelling G, Papassotiropoulos A, et al. Low-dose cortisol for symptoms of posttraumatic stress disorder. Am J Psychiatry. 2004;161:1488-90.

10 Schnurr PP, Green BL (eds.). Trauma and Health: Physical Health Consequences of Exposure to Extreme Stress. Washington, DC: APA; 2004.

11 Foa EB, Keane TM, Friedman MJ. Effective Treatments for PTSD: Practice Guidelines from the International Society for Traumatic Stress Studies. New York: Guilford Press; 2000.

12 Davidson PR, Parker KCH. Eye movement desensitization and reprocessing (EMDR): a meta-analysis. J Consult Clin Psychol. 2001;69:302-16.

13 Schnyder U. Why new psychotherapies for posttraumatic stress disorder? Psychother Psychosom. 2005;74:199-201.

14 Gersons BPR, Carlier IVE, Lamberts RD, van der Kolk BA. Randomized clinical trial of brief eclectic psychotherapy for police officers with posttraumatic stress disorder. J Trauma Stress. 2000; 13:333-48.

15 Neuner F, Schauer M, Karunakara U, Klaschik C, Elbert T. A comparison of Narrative Exposure Therapy, supportive counseling and psychoeducation for treating posttraumatic stress disorder in an African refugee settlement. J Consult Clin Psychol. 2004;72:579-87.

16 Herman JL. Trauma and Recovery. The Aftermath of Violence - from Domestic Abuse to Political Terror. New York: Basic Books; 1992.

17 Reddemann L. Psychodynamisch imaginative Traumatherapie. Das Manual. Stuttgart: Pfeiffer bei Klett-Cotta; 2004. 